\title{
Pacific
}

Journal of

Mathematics

\section{ON THE ATKIN POLYNOMIALS}

Ahmad EL-Guindy AND Mourad E. H. IsmaIL 


\title{
ON THE ATKIN POLYNOMIALS
}

\author{
Ahmad EL-Guindy AND Mourad E. H. IsmaIL
}

\begin{abstract}
We identify the Atkin polynomials in terms of associated Jacobi polynomials. Our identification then takes advantage of the theory of orthogonal polynomials and their asymptotics to establish many new properties of the Atkin polynomials. This shows that corecursive polynomials may lead to interesting sets of orthogonal polynomials.
\end{abstract}

\section{Introduction}

In unpublished work, Oliver Atkin introduced a family of orthogonal polynomials with fascinating number-theoretic properties: They are the unique family of monic orthogonal polynomials corresponding to a unique scalar product on the space of polynomials in the modular $j$-invariant for which all Hecke operators are selfadjoint. Furthermore, their reductions modulo a prime $p \geq 5$ are also very significant in the theory of elliptic curves, as they match the supersingular polynomial at $p$ whenever the degrees agree. For all the number-theoretic definitions, as well as beautiful proofs of these and other facts about the Atkin polynomials, we refer the reader to the excellent [Kaneko and Zagier 1998], where Atkin's results were popularized, simplified, and expanded upon.

The Atkin polynomials are generated by the recurrence relation

$$
\begin{aligned}
A_{n+1}(x)= & {\left[x-24 \frac{144 n^{2}-29}{(2 n+1)(2 n-1)}\right] A_{n}(x) } \\
& -36 \frac{(12 n-13)(12 n-7)(12 n-5)(12 n+1)}{n(n-1)(2 n-1)^{2}} A_{n-1}(x), \quad n>1,
\end{aligned}
$$

through the initial conditions

$$
A_{0}(x)=1, \quad A_{1}(x)=x-720, \quad A_{2}(x)=x^{2}-1640 x+269280 .
$$

Research supported by the DSFP at King Saud University through grant DSFP/MATH 01 and by the NPST program of King Saud University, project number 10-MAT1293-02.

MSC2010: primary 33C47, 11F03; secondary 33C05.

Keywords: associated Jacobi polynomials, asymptotics, weight functions, scaled invariant, modular function, birth and death process polynomials, supersingular polynomials, Hecke operators. 
The polynomials $\left\{A_{n}(x)\right\}$ are orthogonal with respect to an absolutely continuous measure supported on $[0,1728]$ (see Section 7).

In this paper we show that the Atkin polynomials are related to the associated Jacobi polynomials of Wimp [1987] and of Ismail and Masson [1991]. This identification leads to many new properties of the polynomials $\left\{A_{n}(x)\right\}$.

It is worth pointing out that the way the Atkin polynomials are defined, that is, defining $P_{0}(x), P_{1}(x)$ and $P_{2}(x)$, then using a recurrence relation to generate the rest, is not unusual in the literature on orthogonal polynomials. The idea is to start with two monic polynomials, $P_{k}(x)$ and $P_{k+1}(x)$, of degrees $k$ and $k+1$, respectively, with real, simple and interlacing zeros. Then use the division algorithm to generate the monic polynomials $P_{n}(x), 0 \leq n<k$; we are guaranteed to have a sequence of monic orthogonal polynomials $\left\{P_{j}(x): 0 \leq j \leq k+1\right\}$. Now use any three-term recurrence relation of the form

$$
P_{n+1}(x)=\left(x-\alpha_{n}\right) P_{n}(x)+\beta_{n} P_{n-1}(x),
$$

where $\alpha_{n} \in \mathbb{R}$ and $\beta_{n}>0$ for $n>k$, to generate the polynomials $\left\{P_{n}(x)\right\}$ for $n>k+1$. The construction above is referred to as "Wendroff's Theorem" in the orthogonal polynomial literature. The interested reader may consult [Ismail 2009] or [Chihara 1978] for the precise statement and the detailed proof of Wendroff's theorem. This is also related to the concept of corecursive polynomials [Chihara 1978].

In Section 2, we recall some preliminary facts about associated Jacobi polynomials and orthogonal polynomials in general. In Section 3, we obtain a representation of (a scaled version of) the Atkin polynomials as a linear combination of the associated Jacobi polynomials of Wimp [1987] and of Ismail and Masson [1991]. Building on that, we provide an explicit representation of the coefficients of the Atkin polynomials in Section 4, a representation in terms of certain hypergeometric functions and an asymptotic expansion in Section 5, and a generating function identity in Section 6. Lastly, in Section 7 we give an explicit description of the weight function in terms of certain ${ }_{2} F_{1}$ functions.

We shall follow the standard notation for hypergeometric functions and orthogonal polynomials as in [Andrews et al. 1999; Ismail 2009; Luke 1969; Rainville 1960; Szegó 1975]. In particular we use $F\left(\begin{array}{c}a, b \\ c\end{array} \mid z\right)$ to mean to mean ${ }_{2} F_{1}\left(\begin{array}{c}a, b \\ c\end{array} \mid z\right)$.

\section{Preliminaries}

Let $\left\{\lambda_{n}\right\}$ and $\left\{\mu_{n}\right\}$ be the birth and death rates of a birth and death process; that is, $\lambda_{n}>0$ and $\mu_{n+1}>0$ for all $n \geq 0$, with $\mu_{0} \geq 0$. Such a process generates a sequence of orthogonal polynomials through a three-term recurrence relation

$$
-x Q_{n}(x)=\lambda_{n} Q_{n+1}(x)-\left(\lambda_{n}+\mu_{n}\right) Q_{n}(x)+\mu_{n} Q_{n-1}(x), \quad n>0,
$$


with the initial conditions

$$
Q_{0}(x)=1, \quad Q_{1}(x)=\left(\lambda_{0}+\mu_{0}-x\right) / \lambda_{0} .
$$

The corresponding monic polynomials satisfy

$$
x \widetilde{Q}_{n}(x)=\widetilde{Q}_{n+1}+\left(\lambda_{n}+\mu_{n}\right) \widetilde{Q}_{n}(x)-\lambda_{n-1} \mu_{n} \widetilde{Q}_{n-1}(x),
$$

with $\widetilde{Q}_{0}(x)=1, \widetilde{Q}_{1}(x)=x-\lambda_{0}-\mu_{0}$. When $\mu_{0} \neq 0$ there is a second natural birth and death process with birth rates $\left\{\lambda_{n}\right\}$ and death rates $\left\{\tilde{\mu}_{n}\right\}$ with $\tilde{\mu}_{n}=\mu_{n}$ for $n>0$ but $\tilde{\mu}_{0}=0$ [Ismail et al. 1988]. The latter birth and death generate a second family of orthogonal polynomials satisfying (2-1) but with initial conditions $Q_{0}(x)=1, Q_{1}(x)=\left(\lambda_{0}-x\right) / \lambda_{0}$. This observation is due to Ismail, Letessier and Valent [Ismail et al. 1988].

The associated polynomials of $\left\{Q_{n}(x)\right\}$ correspond to the birth and death rates $\left\{\lambda_{n+c}\right\}$ and death rates $\left\{\mu_{n+c}\right\}$, when such rates are well defined. Since we consider $c \geq 0$, usually $\mu_{c}>0$. Thus we usually have two families of associated polynomials. One is defined when $\mu_{c}$ is defined from the pattern of $\mu_{n}$. When $\mu_{c} \neq 0$, a second family arises if $\mu_{n+c}$, when $n=0$ is interpreted as zero.

Recall that the Jacobi polynomials $\left\{P_{n}^{(\alpha, \beta)}(x)\right\}$ can be defined by the three-term recurrence relation

$$
\begin{aligned}
& 2(n+1)(n+\alpha+\beta+1)(\alpha+\beta+2 n) P_{n+1}^{(\alpha, \beta)}(x) \\
& =(\alpha+\beta+2 n+1)\left[\left(\alpha^{2}-\beta^{2}\right)+x(\alpha+\beta+2 n+2)(\alpha+\beta+2 n)\right] P_{n}^{(\alpha, \beta)}(x) \\
& -2(\alpha+n)(\beta+n)(\alpha+\beta+2 n+2) P_{n-1}^{(\alpha, \beta)}(x),
\end{aligned}
$$

for $n \geq 0$, with $P_{-1}^{(\alpha, \beta)}(x)=0, P_{0}^{(\alpha, \beta)}(x)=1$. We now set

$$
\begin{aligned}
V_{n}^{(\alpha, \beta)}(x) & =\frac{n !(\alpha+\beta+1)_{n}}{(\alpha+\beta+1)_{2 n}} P_{n}^{(\alpha, \beta)}(2 x-1) \\
& =\frac{n !}{(n+\alpha+\beta+1)_{n}} P_{n}^{(\alpha, \beta)}(2 x-1) .
\end{aligned}
$$

One can easily verify that the polynomials $\left\{V_{n}^{(\alpha, \beta)}(x)\right\}$ are monic birth and death process polynomials $\widetilde{Q}_{n}$, with rates

$$
\begin{aligned}
& \lambda_{n}=\frac{(n+\beta+1)(n+\alpha+\beta+1)}{(2 n+\alpha+\beta+1)(2 n+\alpha+\beta+2)}, \\
& \mu_{n}=\frac{n(n+\alpha)}{(2 n+\alpha+\beta)(2 n+\alpha+\beta+1)} .
\end{aligned}
$$

Wimp [1987] considered the recurrence relation obtained by formally replacing $n$ by $n+c$ in (2-4), and he showed that the new relation has two linearly independent solutions $P_{n}^{(\alpha, \beta)}(x ; c)$ and $P_{n-1}^{(\alpha, \beta)}(x ; c+1)$. Ismail and Masson [1991] identified 
the birth and death rates corresponding to that three-term recurrence relation and provided two linearly independent solutions $P_{n}^{(\alpha, \beta)}(x ; c)$ and $\mathscr{P}_{n}^{(\alpha, \beta)}(x ; c)$. They then used the notation

$$
R_{n}^{(\alpha, \beta)}(x ; c)=P_{n}^{(\alpha, \beta)}(2 x-1 ; c), \quad \mathscr{R}_{n}^{(\alpha, \beta)}(x ; c)=\mathscr{P}_{n}^{(\alpha, \beta)}(2 x-1 ; c) .
$$

We shall use the notation

$$
\begin{aligned}
& V_{n}^{(\alpha, \beta)}(x ; c)=\frac{(c+1)_{n}(\alpha+\beta+c+1)_{n}}{(\alpha+\beta+2 c+1)_{2 n}} R_{n}^{(\alpha . \beta)}(x ; c), \\
& \mathscr{V}_{n}^{(\alpha, \beta)}(x ; c)=\frac{(c+1)_{n}(\alpha+\beta+c+1)_{n}}{(\alpha+\beta+2 c+1)_{2 n}} \mathscr{R}_{n}^{(\alpha . \beta)}(x ; c) .
\end{aligned}
$$

To lighten our notation, we shall occasionally omit the parameters when the context is clear. We consider the birth and death rates

$$
\begin{aligned}
& \lambda_{n}=\frac{(n+c+\beta+1)(n+c+\alpha+\beta+1)}{(2 n+2 c+\alpha+\beta+1)(2 n+2 c+\alpha+\beta+2)}, \quad n \geq 0, \\
& \mu_{n}=\frac{(n+c)(n+c+\alpha)}{(2 n+2 c+\alpha+\beta)(2 n+2 c+\alpha+\beta+1)}, \quad n>0,
\end{aligned}
$$

with

$$
\mu_{0}:=\left\{\begin{array}{cl}
\frac{c(c+\alpha)}{(2 c+\alpha+\beta)(2 c+\alpha+\beta+1)} & \text { for } V \\
0 & \text { for } \mathcal{V}
\end{array}\right.
$$

\section{The Atkin polynomials}

In order to compare the Atkin polynomials with other results in the literature we need to renormalize them. Let

$$
A_{n}(1728 y)=(1728)^{n} \mathscr{A}_{n}(y) .
$$

The polynomials $\mathscr{A}_{n}$ are now generated by the recurrence

$$
\begin{aligned}
& \mathscr{A}_{n+1}(x) \\
& \quad=\left[x-\frac{2\left(n^{2}-\frac{29}{144}\right)}{4 n^{2}-1}\right] \mathscr{A}_{n}(x)-\frac{\left(n-\frac{13}{12}\right)\left(n-\frac{7}{12}\right)\left(n-\frac{5}{12}\right)\left(n+\frac{1}{12}\right)}{2 n(2 n-1)^{2}(2 n-2)} \mathscr{A}_{n-1}(x)
\end{aligned}
$$

for $n>1$. The initial conditions are

$$
\mathscr{A}_{0}(x)=1, \quad \mathscr{A}_{1}(x)=x-\frac{5}{12}, \quad \mathscr{A}_{2}(x)=x^{2}-\frac{205}{216} x+\frac{935}{10368} .
$$

Kaneko and Zagier [1998] wrote the recurrence relation (1-1) in the monic form (2-3). Indeed their (19), when written in terms of the $\mathscr{A}_{n}$, corresponds to (2-3) with

$$
\lambda_{n}=\frac{\left(n-\frac{1}{12}\right)\left(n+\frac{5}{12}\right)}{2 n(2 n+1)}, \quad \mu_{n}=\frac{\left(n-\frac{5}{12}\right)\left(n+\frac{1}{12}\right)}{2 n(2 n-1)} .
$$


From $(2-8)$, we see that $V_{n}^{(\alpha, \beta)}(x ; c)$ and $\mathscr{V}_{n}^{(\alpha, \beta)}(x ; c)$ satisfy the second-order difference equation

$$
\begin{gathered}
T_{n+1}(x)=\left(x+\frac{\alpha^{2}-\beta^{2}-(2 n+2 c+\alpha+\beta)(2 n+2 c+\alpha+\beta+2)}{2(2 n+2 c+\alpha+\beta)(2 n+2 c+\alpha+\beta+2)}\right) T_{n}(x) \\
-\frac{(n+c)(n+c+\alpha)(n+c+\beta)(n+c+\alpha+\beta)}{(2 n+2 c+\alpha+\beta-1)(2 n+2 c+\alpha+\beta)^{2}(2 n+2 c+\alpha+\beta+1)} T_{n-1}(x)
\end{gathered}
$$

for $n \geq 1$, with the initial conditions $V_{0}=\mathscr{V}_{0}=1$ and

$$
\begin{aligned}
& V_{1}^{(\alpha, \beta)}(x ; c)=x-\left(\lambda_{0}+\mu_{0}\right), \\
& \mathcal{V}_{1}^{(\alpha, \beta)}(x ; c)=x-\lambda_{0},
\end{aligned}
$$

where $\lambda_{n}$ and $\mu_{n}$ are defined as in (2-9)-(2-10). On the other hand, we see that the sequence $\left\{\mathscr{A}_{n+1}(x)\right\}_{n=-1}^{\infty}$ is a solution of the second-order difference equation

$$
\begin{aligned}
& T_{n+1}(x)=\left(x-\frac{7+36(2 n+1)(2 n+3)}{72(2 n+1)(2 n+3)}\right) T_{n}(x) \\
&-\frac{\left(n-\frac{1}{12}\right)\left(n+\frac{5}{12}\right)\left(n+\frac{7}{12}\right)\left(n+\frac{13}{12}\right)}{(2 n)(2 n+1)^{2}(2 n+2)} T_{n-1}(x)
\end{aligned}
$$

for $n \geq 1$. It is not hard to check that (3-7) is identical to (3-5) in exactly four cases, namely,

$$
(\alpha, \beta, c) \in S:=\left\{\left(-\frac{1}{2},-\frac{2}{3}, \frac{13}{12}\right),\left(\frac{1}{2},-\frac{2}{3}, \frac{7}{12}\right),\left(-\frac{1}{2}, \frac{2}{3}, \frac{5}{12}\right),\left(\frac{1}{2}, \frac{2}{3},-\frac{1}{12}\right)\right\} .
$$

Theorem 3.1. For $n \geq 0$ and $(\alpha, \beta, c) \in S$, we have the following representations for $\mathscr{A}_{n+1}(x)$ :

$$
\begin{aligned}
& \mathscr{A}_{n+1}(x)=\left(x-\frac{5}{12}\right) V_{n}^{(\alpha, \beta)}(x ; c)-\frac{91}{384} V_{n-1}^{(\alpha, \beta)}(x ; c+1), \\
& \mathscr{A}_{n+1}(x)=(x-8) V_{n}^{(-1 / 2,2 / 3)}\left(x ; \frac{5}{12}\right)+\frac{91}{12} V_{n}^{(-1 / 2,2 / 3)}\left(x ; \frac{5}{12}\right), \\
& \mathscr{A}_{n+1}(x)=x V_{n}^{(1 / 2,-2 / 3)}\left(x ; \frac{7}{12}\right)-\frac{5}{12} \mathscr{V}_{n}^{(1 / 2,-2 / 3)}\left(x ; \frac{7}{12}\right) .
\end{aligned}
$$

Proof. It is straightforward to check that for any $(\alpha, \beta, c) \in S$,

$$
\left\{V_{n}^{(\alpha, \beta)}(x ; c), \mathscr{V}_{n}^{(\alpha, \beta)}(x ; c)\right\}
$$

is a basis of solutions of (3-7), and the same is true for

$$
\left\{V_{n}^{(\alpha, \beta)}(x ; c), V_{n-1}^{(\alpha, \beta)}(x ; c+1)\right\} .
$$

The results follow by simple linear algebra on the equations corresponding to $n=0$ and $n=1$. 
We note that $V_{n}^{(\alpha, \beta)}(x ; c)$ is the same for the four triples in $S$, whereas we have two possibilities for $\mathcal{V}_{n}^{(\alpha, \beta)}(x ; c)$, depending on whether $\beta=\frac{2}{3}$ or $\beta=-\frac{2}{3}$. For convenience we explicitly write down the first few of these polynomials:

$$
\begin{aligned}
& V_{0}^{(\alpha, \beta)}(x ; c)=1, \\
& V_{1}^{(\alpha, \beta)}(x ; c)=x-\frac{115}{216}, \\
& V_{2}^{(\alpha, \beta)}(x ; c)=x^{2}-\frac{187}{180} x+\frac{11621}{55296} ; \\
& V_{-1}^{(\alpha, \beta)}(x ; c+1)=0 \\
& V_{0}^{(\alpha, \beta)}(x ; c+1)=1 \\
& V_{1}^{(\alpha, \beta)}(x ; c+1)=x-\frac{547}{1080} ; \\
& \mathscr{V}_{0}^{(1 / 2,-2 / 3)}\left(x ; \frac{7}{12}\right)=\mathscr{V}_{0}^{(-1 / 2,-2 / 3)}\left(x ; \frac{13}{12}\right)=1, \\
& \mathscr{V}_{1}^{(1 / 2,-2 / 3)}\left(x ; \frac{7}{12}\right)=\mathscr{V}_{1}^{(-1 / 2,-2 / 3)}\left(x ; \frac{13}{12}\right)=x-\frac{187}{864}, \\
& \mathscr{V}_{2}^{(1 / 2,-2 / 3)}\left(x ; \frac{7}{12}\right)=\mathscr{V}_{2}^{(-1 / 2,-2 / 3)}\left(x ; \frac{13}{12}\right)=x^{2}-\frac{347}{480} x+\frac{124729}{2488320} \\
& \\
& \mathscr{V}_{0}^{(1 / 2,2 / 3)}\left(x ; \frac{-1}{12}\right)=\mathscr{V}_{0}^{(-1 / 2,2 / 3)}\left(x ; \frac{5}{12}\right)=1, \\
& \mathscr{V}_{1}^{(1 / 2,2 / 3)}\left(x ; \frac{-1}{12}\right)=\mathscr{V}_{1}^{(-1 / 2,2 / 3)}\left(x ; \frac{5}{12}\right)=x-\frac{475}{864}, \\
& \mathscr{V}_{2}^{(1 / 2,2 / 3)}\left(x ; \frac{-1}{12}\right)=\mathscr{V}_{2}^{(-1 / 2,2 / 3)}\left(x ; \frac{5}{12}\right)=x^{2}-\frac{169}{160} x+\frac{108965}{497664} .
\end{aligned}
$$

One can check the first few cases of Theorem 3.1 using the equalities

$$
\begin{aligned}
& \mathscr{A}_{1}(x)=x-\frac{5}{12} \\
& \mathscr{A}_{2}(x)=x^{2}-\frac{205}{216} x+\frac{935}{10368}, \\
& \mathscr{A}_{3}(x)=x^{3}-\frac{131}{90} x^{2}+\frac{28277}{55296} x-\frac{124729}{5971968}
\end{aligned}
$$

\section{Explicit representations}

Wimp [1987, p. 987] gave an explicit formula for $R_{n}^{(\alpha, \beta)}(x ; c)$. When translated in terms of the $V_{n}$ polynomials it becomes

$$
\begin{aligned}
V_{n}^{(\alpha, \beta)}(x ; c)=(-1)^{n} & \frac{(c+1)_{n}(\beta+c+1)_{n}}{(\alpha+\beta+2 c+n+1)_{n} n !} \\
\times \sum_{k=0}^{n} \frac{(-n)_{k}(n+2 c+\alpha+\beta+1)_{k}}{(c+1)_{k}(c+\beta+1)_{k}} x^{k} & \quad \times{ }_{4} F_{3}\left(\begin{array}{c}
k-n, n+k+\alpha+\beta+2 c+1, c+\beta, c \\
k+\beta+c+1, k+c+1, \alpha+\beta+2 c
\end{array}\right) .
\end{aligned}
$$


On the other hand, Ismail and Masson [1991, Theorem 3.3] gave a similar formula for $\mathscr{R}_{n}^{(\alpha, \beta)}(x ; c)$, which leads to

$$
\begin{aligned}
& \mathscr{V}_{n}^{(\alpha, \beta)}(x ; c) \\
& =(-1)^{n} \frac{(c+1)_{n}(\beta+c+1)_{n}}{(\alpha+\beta+2 c+n+1)_{n} n !} \\
& \times \sum_{k=0}^{n} \frac{(-n)_{k}(n+2 c+\alpha+\beta+1)_{k}}{(c+1)_{k}(c+\beta+1)_{k}} x^{k} \\
& \quad \times{ }_{4} F_{3}\left(\begin{array}{c}
k-n, n+k+\alpha+\beta+2 c+1, c+\beta+1, c \mid c \\
k+\beta+c+1, k+c+1, \alpha+\beta+2 c+1
\end{array}\right) .
\end{aligned}
$$

The following theorem establishes an analogous representation of $\mathscr{A}_{n+1}(x)$ :

Theorem 4.1. For $n \geq 0$, we have

$$
\begin{aligned}
& \mathscr{A}_{n+1}(x)= \\
& \frac{\left(\frac{19}{12}\right)_{n}\left(\frac{11}{12}\right)_{n}}{(n+2)_{n}(-n)_{n}}
\end{aligned}
$$

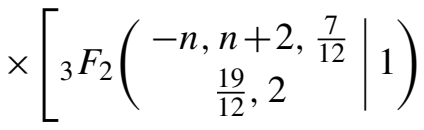

$$
\begin{aligned}
& +\sum_{k=0}^{n} \frac{(-n)_{k}(n+2)_{k}}{\left(\frac{19}{12}\right)_{k}\left(\frac{11}{12}\right)_{k}} x^{k+1}\left\{\frac{6}{5}{ }_{4} F_{3}\left(\begin{array}{c}
k-n, n+k+2, \frac{11}{12}, \frac{-5}{12} \\
k+\frac{11}{12}, k+\frac{19}{12}, 1
\end{array} \mid 1\right)\right.
\end{aligned}
$$

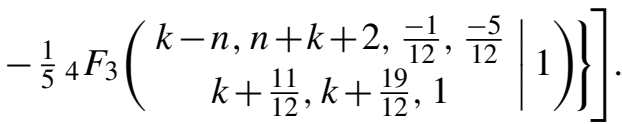

Proof. From (3-10) we have

$$
\mathscr{A}_{n+1}(x)=x V_{n}^{(1 / 2,-2 / 3)}\left(x ; \frac{7}{12}\right)-\frac{5}{12} \mathscr{V}_{n}^{(1 / 2,-2 / 3)}\left(x ; \frac{7}{12}\right), \quad n \geq 0 ;
$$

we see that the coefficient of $x^{k+1}$ in $\mathscr{A}_{n+1}(x)$ is given by

$$
\begin{aligned}
& (-1)^{n} \frac{\left(\frac{19}{12}\right)_{n}\left(\frac{11}{12}\right)_{n}}{(n+2)_{n} n !} \frac{(-n)_{k}(n+2)_{k}}{\left(\frac{19}{12}\right)_{k}\left(\frac{11}{12}\right)_{k}} \\
& \times\left[{ }_{4} F_{3}\left(\begin{array}{c}
k-n, n+k+2, \frac{-1}{12}, \frac{7}{12} \\
k+\frac{11}{12}, k+\frac{19}{12}, 1
\end{array}\right)\right. \\
& \left.-\frac{5}{12} \frac{(k-n)(n+k+2)}{\left(k+\frac{19}{12}\right)\left(k+\frac{11}{12}\right)}{ }_{4} F_{3}\left(\begin{array}{c}
k+1-n, n+k+3, \frac{11}{12}, \frac{7}{12} \\
k+1+\frac{11}{12}, k+1+\frac{19}{12}, 2
\end{array}\right)\right] \text {. }
\end{aligned}
$$


The coefficient of $y^{m}$ in

$$
\begin{aligned}
& { }_{4} F_{3}\left(\begin{array}{c}
k-n, n+k+2, \frac{-1}{12}, \frac{7}{12} \\
k+\frac{11}{12}, k+\frac{19}{12}, 1
\end{array} \mid y\right) \\
& -\frac{5 y}{12} \frac{(k-n)(n+k+2)}{\left(k+\frac{19}{12}\right)\left(k+\frac{11}{12}\right)}{ }_{4} F_{3}\left(\begin{array}{c}
k+1-n, n+k+3, \frac{11}{12}, \frac{7}{12} \\
k+1+\frac{11}{12}, k+1+\frac{19}{12}, 2
\end{array} \mid y\right)
\end{aligned}
$$

is

$$
\begin{aligned}
& \frac{(k-n)_{m}(n+k+2)_{m}\left(\frac{-1}{12}\right)_{m}\left(\frac{7}{12}\right)_{m}}{\left(k+\frac{11}{12}\right)_{m}\left(k+\frac{19}{12}\right)_{m}(m !)^{2}} \\
& \quad-\frac{5 m}{12} \frac{(k-n)(n+k+2)}{\left(k+\frac{19}{12}\right)\left(k+\frac{11}{12}\right)} \frac{(k-n+1)_{m-1}(n+k+3)_{m-1}\left(\frac{11}{12}\right)_{m-1}\left(\frac{7}{12}\right)_{m-1}}{\left(k+1+\frac{11}{12}\right)_{m-1}\left(k+1+\frac{19}{12}\right)_{m-1}(2)_{m-1}(m) !} .
\end{aligned}
$$

Using the identity $(z)_{m}=z(z+1)_{m-1}$, we get that this coefficient is

$$
\begin{aligned}
\frac{(k-n)_{m}(n+k+2)_{m}\left(\frac{-1}{12}\right)_{m}\left(\frac{7}{12}\right)_{m}}{\left(k+\frac{11}{12}\right)_{m}\left(k+\frac{19}{12}\right)_{m}(m !)^{2}}\left(1+\frac{\left(\frac{-5}{12}\right)(-12 m)}{\left(\frac{7}{12}+m-1\right)}\right) & \\
= & \frac{(k-n)_{m}(n+k+2)_{m}\left(\frac{-1}{12}\right)_{m}\left(\frac{-5}{12}\right)_{m}}{\left(k+\frac{11}{12}\right)_{m}\left(k+\frac{19}{12}\right)_{m}(m !)^{2}}\left(1-\frac{72}{5} m\right) \\
= & \frac{1}{5} \frac{(k-n)_{m}(n+k+2)_{m}\left(\frac{-5}{12}\right)_{m}}{\left(k+\frac{11}{12}\right)_{m}\left(k+\frac{19}{12}\right)_{m}(m !)^{2}}\left(6\left(\frac{11}{12}\right)_{m}-\left(\frac{-1}{12}\right)_{m}\right) .
\end{aligned}
$$

In the last equality, we used

$$
\left(\frac{-1}{12}\right)_{m}\left(m-\frac{5}{72}\right)=\left(\frac{-1}{12}\right)_{m}\left[\left(m-\frac{1}{12}\right)+\frac{1}{72}\right]=-\frac{1}{12}\left(\frac{11}{12}\right)_{m}+\frac{1}{72}\left(\frac{-1}{12}\right)_{m} .
$$

It now follows that

$$
\begin{aligned}
& {\left[{ }_{4} F_{3}\left(\begin{array}{c}
k-n, n+k+2, \frac{-1}{12}, \frac{7}{12} \\
k+\frac{11}{12}, k+\frac{19}{12}, 1
\end{array}\right)\right.} \\
& \left.-\frac{5 y}{12} \frac{(k-n)(n+k+2)}{\left(k+\frac{19}{12}\right)\left(k+\frac{11}{12}\right)}{ }_{4} F_{3}\left(\begin{array}{c}
k+1-n, n+k+3, \frac{11}{12}, \frac{7}{12} \\
k+1+\frac{11}{12}, k+1+\frac{19}{12}, 2
\end{array} \mid y\right)\right] \\
& =\frac{6}{5}{ }_{4} F_{3}\left(\begin{array}{c}
k-n, n+k+2, \frac{11}{12}, \frac{-5}{12} \\
k+\frac{11}{12}, k+\frac{19}{12}, 1
\end{array} \mid y\right) \\
& -\frac{1}{5}{ }_{4} F_{3}\left(\begin{array}{c}
k-n, n+k+2, \frac{-1}{12}, \frac{-5}{12} \\
k+\frac{11}{12}, k+\frac{19}{12}, 1
\end{array} \mid y\right) .
\end{aligned}
$$

The result now follows by substituting (4-6) with $y=1$ into (4-4) . 
Remark 4.2. There is another explicit representation of a somewhat different form than (4-3) for the Atkin polynomials. Indeed, it follows from Theorem 4(ii) in [Kaneko and Zagier 1998] that

$$
\mathscr{A}_{n}(x)=\sum_{i=0}^{n} \sum_{m=0}^{i}(-1)^{m}\left(\begin{array}{c}
\frac{-1}{12} \\
i-m
\end{array}\right)\left(\begin{array}{c}
\frac{-5}{12} \\
i-m
\end{array}\right)\left(\begin{array}{c}
n+\frac{1}{12} \\
m
\end{array}\right)\left(\begin{array}{c}
n-\frac{7}{12} \\
m
\end{array}\right)\left(\begin{array}{c}
2 n-1 \\
m
\end{array}\right)^{-1} x^{n-i}
$$

\section{Asymptotics}

Wimp [1987, Proof of Theorem 1] showed that the functions $u_{n}$ and $y_{n}\left(u_{n}\right.$ and $v_{n}$ in his notation) defined by

$$
\begin{aligned}
& u_{n}^{(\alpha, \beta)}(x ; c)=(-1)^{n} \frac{\Gamma(n+\beta+c+1)}{\Gamma(n+c+1)} F\left(\begin{array}{c}
-n-c, n+\alpha+\beta+c+1 \mid x \\
1+\beta
\end{array}\right), \\
& y_{n}^{(\alpha, \beta)}(x ; c)=(-1)^{n} \frac{\Gamma(n+\alpha+c+1)}{\Gamma(n+\alpha+\beta+c+1)} F\left(\begin{array}{c}
-n-\beta-c, n+\alpha+c+1 \\
1-\beta
\end{array} \mid x\right),
\end{aligned}
$$

satisfy the same recurrence relation satisfied by $R_{n}$ and $\mathscr{R}_{n}$, and thus the latter can be represented as linear combinations of the former. We shall slightly modify these functions so as to replace the gamma factors by rising factorials (thus getting rational rather than transcendental coefficients when the parameters are rational) as follows. Set

$$
\begin{aligned}
U_{n}^{(\alpha, \beta)}(x ; c) & =\frac{\Gamma(c+1)}{\Gamma(\beta+c+1)} u_{n}^{(\alpha, \beta)}(x ; c), \\
Y_{n}^{(\alpha, \beta)}(x ; c) & =\frac{\Gamma(\alpha+\beta+c+1)}{\Gamma(\alpha+c+1)} y_{n}^{(\alpha, \beta)}(x ; c) .
\end{aligned}
$$

Thus we have

$$
\begin{aligned}
& U_{n}^{(\alpha, \beta)}(x ; c)=(-1)^{n} \frac{(\beta+c+1)_{n}}{(c+1)_{n}} F\left(\begin{array}{c}
-n-c, n+\alpha+\beta+c+1 \mid x), \\
1+\beta
\end{array}\right) \\
& Y_{n}^{(\alpha, \beta)}(x ; c)=(-1)^{n} \frac{(\alpha+c+1)_{n}}{(\alpha+\beta+c+1)_{n}} F\left(\begin{array}{c}
-n-\beta-c, n+\alpha+c+1 \\
1-\beta
\end{array} \mid x\right) .
\end{aligned}
$$

Note that since the factors multiplied by $u_{n}$ and $y_{n}$ in (5-2) are independent of $n$, $U_{n}$ and $Y_{n}$ satisfy the same recurrence as $R_{n}$ and $\mathscr{R}_{n}$. Indeed, after a simple Kummer transformation, Formula (28) on p. 988 of [Wimp 1987] can be written as

$$
\begin{aligned}
R_{n}=\frac{(\beta+c)(\alpha+\beta+c)}{\beta(\alpha+\beta+2 c)} F\left(\begin{array}{c}
c, 1-(\alpha+\beta+c) \\
1-\beta
\end{array} \mid x\right) U_{n} \\
\quad-\frac{c(\alpha+c)}{\beta(\alpha+\beta+2 c)} F\left(\begin{array}{c}
\beta+c, 1-(\alpha+c) \\
1+\beta
\end{array} \mid x\right) Y_{n} .
\end{aligned}
$$


Similarly, Theorem 3.10 of [Ismail and Masson 1991] leads to

$$
\begin{aligned}
\mathscr{R}_{n}=F\left(\begin{array}{c|c}
c,-(\alpha+\beta+c) \\
-\beta
\end{array} \mid x\right) & U_{n} \\
& \quad-\frac{c(\alpha+c)}{\beta(\beta+1)} x F\left(\begin{array}{c}
1+\beta+c, 1-(\alpha+c) \\
2+\beta
\end{array} \mid x\right) Y_{n} .
\end{aligned}
$$

The following theorem provides the analogous representation for the Atkin polynomials:

Theorem 5.1. Let $U_{n}$ and $Y_{n}$ be as in (5-3), and set

$$
\begin{aligned}
& \widetilde{U}_{n}^{(\alpha, \beta)}(x ; c)=\frac{(c+1)_{n}(\alpha+\beta+c+1)_{n}}{(\alpha+\beta+2 c+1)_{2 n}} U_{n}^{(\alpha, \beta)}(x ; c), \\
& \tilde{Y}_{n}^{(\alpha, \beta)}(x ; c)=\frac{(c+1)_{n}(\alpha+\beta+c+1)_{n}}{(\alpha+\beta+2 c+1)_{2 n}} Y_{n}^{(\alpha, \beta)}(x ; c) .
\end{aligned}
$$

Then we have

$$
\mathscr{A}_{n+1}(x)=C(x) \widetilde{U}_{n}^{(1 / 2,-2 / 3)}\left(x ; \frac{7}{12}\right)+D(x) \widetilde{Y}_{n}^{(1 / 2,-2 / 3)}\left(x ; \frac{7}{12}\right), \quad n \geq 0,
$$

with $C(x)$ and $D(x)$ given by

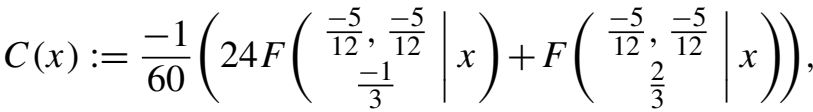

$$
\begin{aligned}
& D(x):=\frac{91}{384} x\left(4 F\left(\begin{array}{c}
\frac{-1}{12}, \frac{-1}{12} \\
\frac{1}{3}
\end{array} \mid x\right)-5 F\left(\begin{array}{c}
\frac{11}{12}, \frac{-1}{12} \\
\frac{4}{3}
\end{array} \mid x\right)\right) \text {. }
\end{aligned}
$$

Proof. From (5-4) and (5-5), we see that

$$
\begin{aligned}
& x R_{n}^{(1 / 2,-2 / 3)}\left(x ; \frac{7}{12}\right)-\frac{5}{12} \mathscr{R}_{n}^{(1 / 2,-2 / 3)}\left(x ; \frac{7}{12}\right)
\end{aligned}
$$

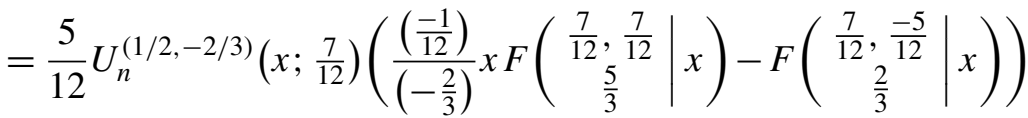

$$
\begin{aligned}
& -x Y_{n}^{(1 / 2,-2 / 3)}\left(x ; \frac{7}{12}\right)\left(\frac{\left(\frac{7}{12}\right)\left(\frac{13}{12}\right)}{\left(-\frac{2}{3}\right)} F\left(\begin{array}{c}
\frac{-1}{12}, \frac{-1}{12} \\
\frac{1}{3}
\end{array} \mid x\right)\right.
\end{aligned}
$$

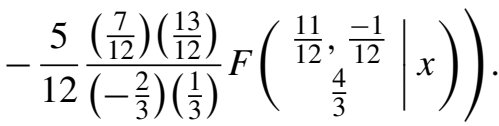

Expanding the hypergeometric series in powers of $x$, we get, after some computation, 
$(5-10) \quad x R_{n}^{(1 / 2,-2 / 3)}\left(x ; \frac{7}{12}\right)-\frac{5}{12} \mathscr{R}_{n}^{(1 / 2,-2 / 3)}\left(x ; \frac{7}{12}\right)$

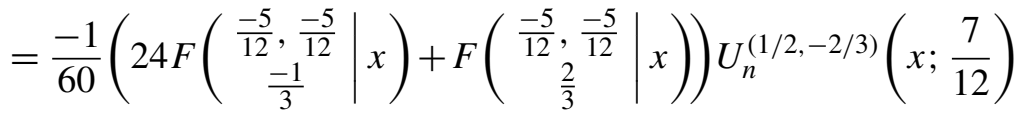

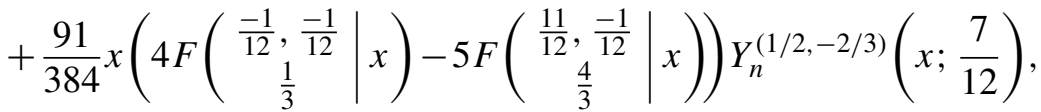

and the result follows from (3-10).

Theorem 5.1 enables us to obtain an asymptotic formula for the Atkin polynomials:

Theorem 5.2. Let $C(x)$ and $D(x)$ be as in (5-8). For fixed $\theta \in(0, \pi / 2)$, the following asymptotic formula holds as $n \rightarrow \infty$ :

(5-11) $\mathscr{A}_{n+1}\left(\sin ^{2} \theta\right)$

$$
\begin{aligned}
\sim \frac{(-1)^{n}}{2^{2 n+1}(\cos \theta)(\sin \theta)^{\frac{7}{6}}} C\left(\sin ^{2} \theta\right) \frac{\Gamma\left(\frac{1}{3}\right)(\sin \theta)^{\frac{2}{3}}}{\Gamma\left(\frac{11}{12}\right) \Gamma\left(\frac{17}{12}\right)} \cos \left[2(n-1) \theta+\frac{\pi}{12}\right] \\
+D\left(\sin ^{2} \theta\right) \frac{\Gamma\left(\frac{5}{3}\right)}{\Gamma\left(\frac{13}{12}\right) \Gamma\left(\frac{19}{12}\right)} \cos \left[2(n-1) \theta-\frac{7 \pi}{12}\right]
\end{aligned}
$$

Proof. We start by recalling the following asymptotic formula, due to Watson, [Luke 1969, (8), p. 237] (all of our asymptotic formulas will be as $n \rightarrow \infty$ ).

$$
\begin{aligned}
& F\left(\begin{array}{c|c}
b-n, n+a & \sin ^{2} \theta \\
d &
\end{array}\right) \\
& \sim \frac{\Gamma(d) n^{-d+\frac{1}{2}}}{\sqrt{\pi}} \frac{(\cos \theta)^{d-a-b-\frac{1}{2}}}{(\sin \theta)^{d-\frac{1}{2}}} \cos \left[2 n \theta+(a-b) \theta-\frac{\pi}{2}\left(d-\frac{1}{2}\right)\right]
\end{aligned}
$$

for fixed $\theta \in(0, \pi)$. Note that Stirling's formula can be written as

$$
\Gamma(n+a) \sim \sqrt{2 \pi} n^{n+a-\frac{1}{2}} e^{-n} \quad \text { as } n \rightarrow \infty,
$$

from which we deduce

$$
\frac{\Gamma(n+a)}{\Gamma(n+b)} \sim n^{a-b} .
$$

Hence

$$
\begin{aligned}
& u_{n}\left(\sin ^{2} \theta\right) \sim \frac{(-1)^{n} \Gamma(1+\beta)(\cos \theta)^{-\alpha-\frac{1}{2}}}{\sqrt{\pi n}(\sin \theta)^{\beta+\frac{1}{2}}} \cos \left[2 n \theta-(\alpha+\beta+2 c+1) \theta-\frac{\pi}{2}\left(\beta+\frac{1}{2}\right)\right], \\
& y_{n}\left(\sin ^{2} \theta\right) \sim \frac{(-1)^{n} \Gamma(1-\beta)(\cos \theta)^{-\alpha-\frac{1}{2}}}{\sqrt{\pi n}(\sin \theta)^{-\beta+\frac{1}{2}}} \cos \left[2 n \theta-(\alpha+\beta+2 c+1) \theta+\frac{\pi}{2}\left(\beta-\frac{1}{2}\right)\right] .
\end{aligned}
$$


Also, from (5-13) we get

$$
\begin{aligned}
& \frac{(c+1)_{n}(\alpha+\beta+c+1)_{n}}{(\alpha+\beta+2 c+1)_{2 n}} \\
& \quad=\frac{\Gamma(\alpha+\beta+2 c+1)}{\Gamma(c+1) \Gamma(\alpha+\beta+c+1)} \frac{\Gamma(n+c+1) \Gamma(n+\alpha+\beta+c+1)}{\Gamma(2 n+\alpha+\beta+2 c+1)} \\
& \quad \sim \frac{\Gamma(\alpha+\beta+2 c+1)}{\Gamma(c+1) \Gamma(\alpha+\beta+c+1)} \sqrt{\pi n}\left(\frac{1}{2}\right)^{2 n+\alpha+\beta+2 c}
\end{aligned}
$$

Substituting $(\alpha, \beta, c)=\left(\frac{1}{2}, \frac{-2}{3}, \frac{7}{12}\right)$, we see that

$$
\begin{aligned}
& \widetilde{U}_{n}\left(\sin ^{2} \theta\right) \sim \frac{(-1)^{n} \Gamma\left(\frac{1}{3}\right)(\sin \theta)^{1 / 6}}{2^{2 n+1} \cos \theta \Gamma\left(\frac{11}{12}\right) \Gamma\left(\frac{17}{12}\right)} \cos \left[2(n-1) \theta+\frac{\pi}{12}\right], \\
& \widetilde{Y}_{n}\left(\sin ^{2} \theta\right) \sim \frac{(-1)^{n} \Gamma\left(\frac{5}{3}\right)(\sin \theta)^{-7 / 6}}{2^{2 n+1} \cos \theta \Gamma\left(\frac{13}{12}\right) \Gamma\left(\frac{19}{12}\right)} \cos \left[2(n-1) \theta-\frac{7 \pi}{12}\right],
\end{aligned}
$$

and the result follows from (5-7) and (5-8).

\section{Generating functions}

We start by recalling a remarkable identity of Flensted-Jensen and Koornwinder [1973]. The interested reader could also consult [Wimp 1987] for more details on various other authors who presented variants of this identity as well as other proofs.

Lemma 6.1. Let $t, x, a, b, d$ be complex numbers with $x \notin[1, \infty)$ and

$$
|t|<\frac{1}{|\sqrt{x}+\sqrt{x-1}|^{2}} .
$$

Then

$$
\begin{aligned}
& \sum_{n=0}^{\infty} \frac{(d+a)_{n}(b)_{n}}{(a+b+1)_{n}} F\left(\begin{array}{c}
-n-a, n+b \\
d
\end{array} \mid x\right) \frac{(-t)^{n}}{n !} \\
& =\left(\frac{z_{2}-t}{z_{2}+t}\right)^{a+d}\left(\frac{2}{z_{2}-t}\right)^{b} F\left(\begin{array}{c|c}
-a, b & t+z_{1} \\
d & \frac{2 t}{2 t}
\end{array}\right) F\left(\begin{array}{c|c}
a+d, a+1 & 2 t \\
a+b+1 & t+z_{2}
\end{array}\right),
\end{aligned}
$$

where $z_{1}=1-\sqrt{(1+t)^{2}-4 x t}$ and $z_{2}=1+\sqrt{(1+t)^{2}-4 x t}$.

To simplify notation we shall write, for $t \neq 0$,

$$
\begin{aligned}
& \delta=\frac{t+z_{1}}{2 t}=\frac{(1+t)-\sqrt{(1+t)^{2}-4 x t}}{2 t}, \\
& \epsilon=\frac{t+z_{2}}{2 t}=\frac{(1+t)+\sqrt{(1+t)^{2}-4 x t}}{2 t} .
\end{aligned}
$$


We clearly have

$$
t(y-\delta)(y-\epsilon)=t y^{2}-(1+t) y+x .
$$

Obviously $z_{2}+t=2 t \epsilon$, and we also have $z_{2}-t=2 t(\epsilon-1)$. Furthermore we have $\delta \epsilon=x / t$. Thus we can rewrite (6-2) for $x \neq 0$ as

$$
\begin{aligned}
\sum_{n=0}^{\infty} \frac{(d+a)_{n}(b)_{n}}{(a+b+1)_{n}} & F\left(\begin{array}{c}
-n-a, n+b \mid x) \frac{(-t)^{n}}{n !} \\
d
\end{array}\right. \\
& =\frac{(x-t \delta)^{a+d-b} \delta^{b}}{x^{a+d}} F\left(\begin{array}{c}
-a, b \\
d
\end{array} \mid \delta\right) F\left(\begin{array}{c}
a+d, a+1 \\
a+b+1
\end{array} \mid \frac{t}{x} \delta\right) .
\end{aligned}
$$

The following proposition provides a generating function for $U_{n}$ and $Y_{n}$ :

Proposition 6.2. Let $U_{n}$ and $Y_{n}$ be as in (5-2). Let $t$ and $x$ be such that $x \notin[1, \infty)$ is nonzero and $\left|t(\sqrt{x}+\sqrt{x-1})^{2}\right|<1$, and set $\delta$ as in (6-3). Then:

$$
\begin{aligned}
& \sum_{n=0}^{\infty} \frac{(\alpha+\beta+c+1)_{n}(c+1)_{n}}{(\alpha+\beta+2 c+2)_{n}} U_{n}(x) \frac{t^{n}}{n !} \\
& =\frac{\delta^{\alpha+\beta+c+1}}{x^{\beta+c+1}(x-t \delta)^{\alpha}} F\left(\begin{array}{c|c}
-c, \alpha+\beta+c+1 \\
1+\beta
\end{array} \mid \delta\right) F\left(\begin{array}{c|c}
\beta+c+1, c+1 & t \delta \\
\alpha+\beta+2 c+2 & \frac{x}{x}
\end{array}\right), \\
& \sum_{n=0}^{\infty} \frac{(\alpha+\beta+c+1)_{n}(c+1)_{n}}{(\alpha+\beta+2 c+2)_{n}} Y_{n}(x) \frac{t^{n}}{n !} \\
& =\frac{\delta^{\alpha+c+1}}{x^{c+1}(x-t \delta)^{\alpha}} F\left(\begin{array}{c|c}
-\beta-c, \alpha+c+1 & \delta \\
1-\beta & \delta
\end{array}\right) F\left(\begin{array}{c|c}
c+1, \beta+c+1 & t \delta \\
\alpha+\beta+2 c+2 & \frac{x}{x}
\end{array}\right) .
\end{aligned}
$$

Proof. From (5-1), we see that

$$
\begin{aligned}
\frac{\Gamma(c+1)}{\Gamma(\beta+c+1)} & \frac{(\alpha+\beta+c+1)_{n}(c+1)_{n}}{(\alpha+\beta+2 c+2)_{n}} u_{n} \\
= & \frac{(c+\beta+1)_{n}(\alpha+\beta+c+1)_{n}}{(\alpha+\beta+2 c+2)_{n}} F\left(\begin{array}{c}
-n-c, n+\alpha+\beta+c+1 \\
1+\beta
\end{array} \mid x\right)
\end{aligned}
$$

and

$$
\begin{aligned}
\frac{\Gamma(\alpha+\beta+c+1)}{\Gamma(\alpha+c+1)} & \frac{(\alpha+\beta+c+1)_{n}(c+1)_{n}}{(\alpha+\beta+2 c+2)_{n}} y_{n} \\
& =\frac{(c+1)_{n}(\alpha+c+1)_{n}}{(\alpha+\beta+2 c+2)_{n}} F\left(\begin{array}{c}
-n-\beta-c, n+\alpha+c+1 \\
1-\beta
\end{array} \mid x\right) .
\end{aligned}
$$

The identities (6-6) and (6-7) follow from applying (6-5) with the choices $(a, b, d)=$ $(c, \alpha+\beta+c+1, \beta+1)$ and $(a, b, d)=(\beta+c, \alpha+c+1,1-\beta)$, respectively. 
Remark 6.3. The result in Proposition 6.2 is essentially due to Wimp. However, we take this opportunity to correct a misprint in the statement of Theorem 5 in [Wimp 1987]: In the first line of page 999, the parameter " $\gamma+c+\beta$ " should be replaced by " $\gamma+c-\beta$ " (in our notation, the later is $\alpha+c+1$ while the former would be $\alpha+c+1+2 \beta$, which indeed doesn't ever seem to figure in the theory).

We next obtain a generating function identity for the Atkin polynomials scaled by a rather unexpected appearance of the Catalan numbers. The right-hand side of the generating series has four summands; each is up to a relatively simple multiple a product of three hypergeometric functions in the variables $x, \delta$ and $1 / \epsilon=t \delta / x$.

Theorem 6.4. Let $C(x)$ and $D(x)$ be as in (5-8), and $\delta$ as in (6-3). Furthermore, let $\left\{C_{n}=1 /(n+1)\left(\begin{array}{c}2 n \\ n\end{array}\right)\right\}_{n}$ denote the sequence of Catalan numbers.

(1) For $0<x<1$ and $|t|<1$, we have

$$
\begin{aligned}
\sum_{n=0}^{\infty} C_{n+1} \mathscr{A}_{n+1}(x) t^{n} & =\frac{\delta^{17 / 12}}{x^{11 / 12} \sqrt{x-t \delta}} F\left(\begin{array}{c}
\frac{11}{12}, \frac{19}{12} \\
3
\end{array} \mid \frac{t \delta}{x}\right) \\
\times & {\left[C(x) F\left(\begin{array}{c}
\frac{-7}{12}, \frac{17}{12} \\
\frac{1}{3}
\end{array} \mid \delta\right)+D(x)\left(\frac{x}{\delta}\right)^{2 / 3} F\left(\begin{array}{c}
\frac{1}{12}, \frac{25}{12} \\
\frac{5}{3}
\end{array} \mid \delta\right)\right] . }
\end{aligned}
$$

(2) For $|t|<1$, we have

$$
\sum_{n=0}^{\infty} C_{n+1} A_{n+1}(0)(-t)^{n}=\frac{-5}{12} F\left(\begin{array}{c}
\frac{11}{12}, \frac{17}{12} \\
3
\end{array} \mid t\right),
$$

and consequently for $n \geq 0$, we have

$$
\mathscr{A}_{n+1}(0)=(-1)^{n}\left(\frac{-5}{12}\right) \frac{\left(\frac{11}{12}\right)_{n}\left(\frac{17}{12}\right)_{n}}{(2 n+1) !} .
$$

Proof. Note that for $0 \leq x<1$, we have

$$
|\sqrt{x}+\sqrt{x-1}|^{2}=|\sqrt{x}+i \sqrt{1-x}|^{2}=1,
$$

so (6-1) indeed translates into $|t|<1$. Now, using (5-6), we see that

$$
\begin{aligned}
\frac{(\alpha+\beta+c+1)_{n}(c+1)_{n}}{(\alpha+\beta+2 c+2)_{n}} U_{n} & =\frac{(\alpha+\beta+2 c+1)_{2 n}}{(\alpha+\beta+2 c+2)_{n}} \widetilde{U}_{n} \\
& =(\alpha+\beta+2 c+1) \frac{(\alpha+\beta+2 c+1+n)_{n}}{(\alpha+\beta+2 c+1+n)} \widetilde{U}_{n},
\end{aligned}
$$

with a similar identity for $Y_{n}$, and (6-10) now follows from (5-7) and Proposition 6.2 by substituting $(\alpha, \beta, c)=\left(\frac{1}{2}, \frac{-2}{3}, \frac{7}{12}\right)$.

When $x=0$ and $|t|<1$, then, in the notation of (6-2), we have $t+z_{1}=0$ and $z_{2}+t=2(1+t)$, and hence $z_{2}-t=2$. Furthermore, we have $C(0)=\frac{-5}{12}$ and 
$D(0)=0$. It thus follows from (6-2) and (5-7) that

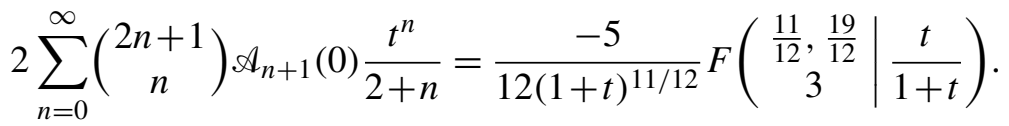

Replacing $t$ with $(-t)$ and applying the Pfaff-Kummer transformation [Erdélyi et al. 1953, Formula (2) on p. 105], we obtain (6-11), from which (6-12) follows by comparing coefficients and simplifying.

Remark 6.5. Formula (6-12) can also be obtained directly from the defining recursion of the Atkin polynomials, as in Proposition 6 of [Kaneko and Zagier 1998]. In that same proposition, and again using only the defining recurrence (1-1), Kaneko and Zagier also obtain a formula equivalent to

$$
\mathscr{A}_{n+1}(1)=\frac{7}{12} \frac{\left(\frac{11}{12}\right)_{n}\left(\frac{19}{12}\right)_{n}}{(2 n+1) !} .
$$

Taking a hint from (6-11), it is straightforward to prove directly from (6-15) that for $|t|<1$, we have

$$
\sum_{n=0}^{\infty} C_{n+1} \mathscr{A}_{n+1}(1) t^{n}=\frac{7}{12} F\left(\begin{array}{c|c}
\frac{11}{12}, \frac{19}{12} & t \\
3 & t
\end{array}\right)
$$

Alternatively, one can prove (6-16) in a manner similar to (6-11), bearing in mind that we have $C(1)=D(1)=0$, whereas $\widetilde{U}_{n}^{(1 / 2,-2 / 3)}\left(x ; \frac{7}{12}\right)$ and $\widetilde{V}_{n}^{(1 / 2,-2 / 3)}\left(x ; \frac{7}{12}\right)$ have simple poles at $x=1$, and thus their product is to be interpreted in the limit $x \rightarrow 1^{-}$as the derivative of the former multiplied by the residue of the latter.

\section{The weight function for the Atkin polynomials}

Kaneko and Zagier [1998] gave the weight function for the Atkin polynomials $A_{n}(j)$ on $[0,1728]$ as

$$
w(j)=\frac{6}{\pi} \theta^{\prime}(j),
$$

where $\theta:[0,1728] \rightarrow[\pi / 3, \pi / 2]$ is the inverse of the monotone increasing function $\theta \mapsto j\left(e^{i \theta}\right)$, where $j(\tau)$ is the usual modular $j$-invariant from the theory of modular forms. In this section we derive an explicit description of the weight function in terms of hypergeometric series. Formula (25) on p. 20 of [Erdélyi et al. 1953] states that an inverse for the scaled $j$-invariant given by

$$
J(z)=\frac{j(z)}{1728}
$$


is obtainable by the formula

$$
z=e^{2 \pi i / 3} \frac{F-\lambda e^{i \pi / 3} J^{1 / 3} F^{*}}{F-\lambda e^{-i \pi / 3} J^{1 / 3} F^{*}},
$$

where

$$
\begin{gathered}
F(J)={ }_{2} F_{1}\left(\begin{array}{c}
\frac{1}{12}, \frac{1}{12} \\
\frac{2}{3}
\end{array} \mid J\right), \\
F^{*}(J)={ }_{2} F_{1}\left(\begin{array}{c}
\frac{5}{12}, \frac{5}{12} \\
\frac{4}{3}
\end{array}\right), \\
\lambda=\frac{\Gamma\left(\frac{2}{3}\right) \Gamma\left(\frac{5}{12}\right) \Gamma\left(\frac{11}{12}\right)}{\Gamma\left(\frac{4}{3}\right) \Gamma\left(\frac{1}{12}\right) \Gamma\left(\frac{7}{12}\right)}=(2-\sqrt{3}) \frac{\Gamma\left(\frac{2}{3}\right) \Gamma^{2}\left(\frac{11}{12}\right)}{\Gamma\left(\frac{4}{3}\right) \Gamma^{2}\left(\frac{7}{12}\right)} .
\end{gathered}
$$

We must note that this is one inverse of many as $J$ is invariant under modular transformations. This particular formula gives, easily, that $z(0)=e^{2 \pi i / 3}$. In order to use the same intervals as in [Kaneko and Zagier 1998], we consider another inverse, corresponding to applying $z \mapsto-1 / z$, thus obtaining

$$
z(J)=e^{\pi i / 3} \frac{F-\lambda e^{-i \pi / 3} J^{1 / 3} F^{*}}{F-\lambda e^{i \pi / 3} J^{1 / 3} F^{*}} .
$$

It is straightforward to verify that using (7-4), we get $z(0)=e^{\pi i / 3}$ and $z(1)=e^{\pi i / 2}$. For $0 \leq J \leq 1, F$ and $F^{*}$ are computed in terms of the converging hypergeometric series and hence are real. Thus in the ratio

$$
\frac{F(J)-\lambda e^{-i \pi / 3} J^{1 / 3} F^{*}(J)}{F(J)-\lambda e^{i \pi / 3} J^{1 / 3} F^{*}(J)}
$$

the denominator is the complex conjugate of the numerator. Hence the ratio has absolute value equal to 1 , and is of the form $e^{i \rho}$. We will show below that $0 \leq \rho \leq \pi / 6$. Thus an explicit description of the function $\theta(j):[0,1728] \rightarrow[\pi / 3, \pi / 2]$ is given by $\theta(j)=\phi(j / 1728)$, where $\phi(J):[0,1] \rightarrow[\pi / 3, \pi / 2]$ is defined by

$$
\phi(J)=\frac{\pi}{3}-i \log \left(\frac{F(J)-\lambda e^{-i \pi / 3} J^{1 / 3} F^{*}(J)}{F(J)-\lambda e^{i \pi / 3} J^{1 / 3} F^{*}(J)}\right)=\frac{\pi}{3}+\rho(J),
$$

and we have

$$
\begin{aligned}
\phi^{\prime}(J) & =-i \frac{F(J)-\lambda e^{i \pi / 3} J^{1 / 3} F^{*}(J)}{F(J)-\lambda e^{-i \pi / 3} J^{1 / 3} F^{*}(J)}\left(\frac{F(J)-\lambda e^{-i \pi / 3} J^{1 / 3} F^{*}(J)}{F(J)-\lambda e^{i \pi / 3} J^{1 / 3} F^{*}(J)}\right)^{\prime} \\
& =-i \frac{W(J)}{\left|F(J)-\lambda e^{-i \pi / 3} J^{1 / 3} F^{*}(J)\right|^{2}},
\end{aligned}
$$

where $W(J)$ is given explicitly by 
(7-6) $\quad W(J)$

$$
\begin{aligned}
= & \left(F(J)-\lambda e^{i \pi / 3} J^{1 / 3} F^{*}(J)\right)\left(F^{\prime}(J)-\lambda e^{-i \pi / 3} J^{1 / 3}\left(F^{*}\right)^{\prime}(J)-\frac{\lambda}{3} e^{-i \pi / 3} J^{-2 / 3} F^{*}(J)\right) \\
& -\left(F(J)-\lambda e^{-i \pi / 3} J^{1 / 3} F^{*}(J)\right)\left(F^{\prime}(J)-\lambda e^{i \pi / 3} J^{1 / 3}\left(F^{*}\right)^{\prime}(J)-\frac{\lambda}{3} e^{i \pi / 3} J^{-2 / 3} F^{*}(J)\right) \\
= & \frac{\lambda}{3} J^{-2 / 3} i \sqrt{3}\left(F(J) F^{*}(J)+3 J F(J)\left(F^{*}\right)^{\prime}(J)-3 J F^{\prime}(J) F^{*}(J)\right) .
\end{aligned}
$$

We also note that $W$ is the Wronskian of two linearly independent solutions for the equation

$$
z(1-z) \frac{d^{2} u}{d z^{2}}+(c-(1+a+b) z) \frac{d u}{d z}-a b u=0,
$$

where here $a=b=\frac{1}{12}$ and $c=\frac{2}{3}$. It follows that $W$ itself satisfies the equation

$$
z(1-z) \frac{d W}{d z}=((a+b+1) z-c) W .
$$

On the open interval $(0,1),(7-7)$ has solution

$$
W(J)=B J^{-2 / 3}(1-J)^{-1 / 2} .
$$

To determine the constant $B$ we compare the coefficient of $J^{-2 / 3}$ in (7-8) and (7-6) to get

$$
B=\frac{i \lambda}{\sqrt{3}}
$$

Hence

$$
\phi^{\prime}(J)=\frac{\lambda}{\sqrt{3}} \frac{J^{-2 / 3}(1-J)^{-1 / 2}}{\left|F(J)-\lambda e^{-i \pi / 3} J^{1 / 3} F^{*}(J)\right|^{2}} .
$$

The fact that the derivative is positive for $0 \leq J \leq 1$ implies that $\phi(J)$ is monotone increasing, and hence that it is bounded between $\phi(0)$ and $\phi(1)$, as we claimed above.

Note that

$$
\begin{aligned}
w(j) & =\frac{6}{\pi} \theta^{\prime}(j)=\frac{6}{1728 \pi} \phi^{\prime}\left(\frac{j}{1728}\right) \\
& =\frac{6 \lambda}{1728 \pi \sqrt{3}} \frac{12\left(12^{2} j^{-2 / 3}\right)\left((1728-j)^{-1 / 2} 12^{3 / 2}\right)}{\left|12 F\left(\frac{j}{1728}\right)-\lambda e^{-i \pi / 3} j^{1 / 3} F^{*}\left(\frac{j}{1728}\right)\right|^{2}} .
\end{aligned}
$$

We have thus proved the following theorem:

Theorem 7.1. Let $\lambda$ be as in (7-3). Then the normalized weight function for the Atkin polynomials $A_{n}(j)$ on the interval $[0,1728]$ is given by

$$
(7-11) \quad w(j)=\frac{144 \lambda}{\pi} \frac{j^{-2 / 3}(1728-j)^{-1 / 2}}{\left|12 F\left(\frac{1}{12}, \frac{1}{\frac{2}{3}} \mid \frac{j}{1728}\right)-\lambda e^{-i \pi / 3} j^{1 / 3} F\left(\frac{5}{12}, \frac{5}{\frac{4}{3}} \mid \frac{j}{1728}\right)\right|^{2}} .
$$




\section{Acknowledgements}

Mourad Ismail wishes to acknowledge the hospitality of Texas A\&M University at Qatar during his visit in September 2012, when this work started. Both authors thank Richard Askey, Jet Foncannon (Wimp), Masanobu Kaneko, and Don Zagier for their interest in this work and for very helpful hints. They are also grateful for the referee for constructive remarks and for suggesting a number of additional references related to the paper.

\section{References}

[Andrews et al. 1999] G. E. Andrews, R. Askey, and R. Roy, Special functions, Encyclopedia of Mathematics and its Applications 71, Cambridge University Press, 1999. MR 2000g:33001 Zbl 0920.33001

[Chihara 1978] T. S. Chihara, An introduction to orthogonal polynomials, Mathematics and its Applications 13, Gordon and Breach, New York, 1978. MR 58 \#1979 Zbl 0389.33008

[Erdélyi et al. 1953] A. Erdélyi, W. Magnus, F. Oberhettinger, and F. G. Tricomi, Higher transcendental functions, I, McGraw-Hill, New York, 1953. MR 15,419i Zbl 0051.30303

[Flensted-Jensen and Koornwinder 1973] M. Flensted-Jensen and T. Koornwinder, "The convolution structure for Jacobi function expansions", Ark. Mat. 11 (1973), 245-262. MR 49 \#5688 Zbl 0267.42009

[Ismail 2009] M. E. H. Ismail, Classical and quantum orthogonal polynomials in one variable, Encyclopedia of Mathematics and its Applications 98, Cambridge University Press, 2009. MR 2010i:33001 Zbl 1172.42008

[Ismail and Masson 1991] M. E. H. Ismail and D. R. Masson, "Two families of orthogonal polynomials related to Jacobi polynomials", pp. 359-375 in Proceedings of the U.S.-Western Europe Regional Conference on Padé Approximants and Related Topics (Boulder, CO, 1988), vol. 21, 1991. MR 93a:33018 Zbl 0744.33004

[Ismail et al. 1988] M. E. H. Ismail, J. Letessier, and G. Valent, "Linear birth and death models and associated Laguerre and Meixner polynomials", J. Approx. Theory 55:3 (1988), 337-348. MR 89m:60204 Zbl 0656.60092

[Kaneko and Zagier 1998] M. Kaneko and D. Zagier, "Supersingular $j$-invariants, hypergeometric series, and Atkin's orthogonal polynomials", pp. 97-126 in Computational perspectives on number theory (Chicago, IL, 1995), edited by D. A. Buell and J. T. Teitelbaum, AMS/IP Stud. Adv. Math. 7, Amer. Math. Soc., Providence, RI, 1998. MR 99b:11064 Zbl 0955.11018

[Luke 1969] Y. L. Luke, The special functions and their approximations, I, Mathematics in Science and Engineering 53, Academic Press, New York, 1969. MR 39 \#3039 Zbl 0193.01701

[Rainville 1960] E. D. Rainville, Special functions, Macmillan, New York, 1960. MR 21 \#6447 Zbl 0092.06503

[Szegô 1975] G. Szegô, Orthogonal polynomials, 4th ed., Colloquium Publications 23, Amer. Math. Soc., Providence, R.I., 1975. MR 51 \#8724 Zbl 0305.42011

[Wimp 1987] J. Wimp, "Explicit formulas for the associated Jacobi polynomials and some applications”, Canad. J. Math. 39:4 (1987), 983-1000. MR 88k:33023 Zbl 0643.33009

Received April 14, 2013. Revised September 9, 2013. 
AHMAD EL-GUINDY

DEPARTMENT OF MATHEMATICS

FACULTY OF SCIENCE

CAIRO UNIVERSITY

GIZA 12613

EGYPT

Current address:

SCIENCE PROGRAM

TEXAS A\&M UNIVERSITY AT QATAR

DOHA, 23874

QATAR

a.elguindy@gmail.com

Mourad E. H. ISMAIL

DEPARTMENT OF MATHEMATICS

UNIVERSITY OF CENTRAL FLORIDA

ORLANDO, FL 32828

UNITED STATES

and

DEPARTMENT OF MATHEMATICS

KING SAUD UNIVERSITY

RIYADH

SAUDI ARABIA

mourad.eh.ismail@gmail.com 


\title{
PACIFIC JOURNAL OF MATHEMATICS
}

\author{
msp.org/pjm
}

Founded in 1951 by E. F. Beckenbach (1906-1982) and F. Wolf (1904-1989)

\section{EDITORS}

Don Blasius (Managing Editor)

Department of Mathematics

University of California

Los Angeles, CA 90095-1555

blasius@math.ucla.edu

\author{
Paul Balmer \\ Department of Mathematics \\ University of California \\ Los Angeles, CA 90095-1555 \\ balmer@math.ucla.edu \\ Robert Finn \\ Department of Mathematics \\ Stanford University \\ Stanford, CA 94305-2125 \\ finn@math.stanford.edu \\ Sorin Popa \\ Department of Mathematics \\ University of California \\ Los Angeles, CA 90095-1555 \\ popa@math.ucla.edu
}

\author{
Vyjayanthi Chari \\ Department of Mathematics \\ University of California \\ Riverside, CA 92521-0135 \\ chari@math.ucr.edu \\ Kefeng Liu \\ Department of Mathematics \\ University of California \\ Los Angeles, CA 90095-1555 \\ liu@math.ucla.edu \\ Jie Qing \\ Department of Mathematics \\ University of California \\ Santa Cruz, CA 95064 \\ qing@ cats.ucsc.edu
}

\section{PRODUCTION}

Silvio Levy, Scientific Editor, production@msp.org

\section{SUPPORTING INSTITUTIONS}

ACADEMIA SINICA, TAIPEI

CALIFORNIA INST. OF TECHNOLOGY

INST. DE MATEMÁTICA PURA E APLICADA

KEIO UNIVERSITY

MATH. SCIENCES RESEARCH INSTITUTE

NEW MEXICO STATE UNIV.

OREGON STATE UNIV.

\author{
STANFORD UNIVERSITY \\ UNIV. OF BRITISH COLUMBIA \\ UNIV. OF CALIFORNIA, BERKELEY \\ UNIV. OF CALIFORNIA, DAVIS \\ UNIV. OF CALIFORNIA, LOS ANGELES \\ UNIV. OF CALIFORNIA, RIVERSIDE \\ UNIV. OF CALIFORNIA, SAN DIEGO \\ UNIV. OF CALIF., SANTA BARBARA
}

\author{
Daryl Cooper \\ Department of Mathematics \\ University of California \\ Santa Barbara, CA 93106-3080 \\ cooper@math.ucsb.edu \\ Jiang-Hua Lu \\ Department of Mathematics \\ The University of Hong Kong \\ Pokfulam Rd., Hong Kong \\ jhlu@maths.hku.hk \\ Paul Yang \\ Department of Mathematics \\ Princeton University \\ Princeton NJ 08544-1000 \\ yang@math.princeton.edu
}

These supporting institutions contribute to the cost of publication of this Journal, but they are not owners or publishers and have no responsibility for its contents or policies.

See inside back cover or msp.org/pjm for submission instructions.

The subscription price for 2014 is US $\$ 410 /$ year for the electronic version, and \$535/year for print and electronic.

Subscriptions, requests for back issues and changes of subscribers address should be sent to Pacific Journal of Mathematics, P.O. Box 4163, Berkeley, CA 94704-0163, U.S.A. The Pacific Journal of Mathematics is indexed by Mathematical Reviews, Zentralblatt MATH, PASCAL CNRS Index, Referativnyi Zhurnal, Current Mathematical Publications and Web of Knowledge (Science Citation Index).

The Pacific Journal of Mathematics (ISSN 0030-8730) at the University of California, c/o Department of Mathematics, 798 Evans Hall \#3840, Berkeley, CA 94720-3840, is published twelve times a year. Periodical rate postage paid at Berkeley, CA 94704, and additional mailing offices. POSTMASTER: send address changes to Pacific Journal of Mathematics, P.O. Box 4163, Berkeley, CA 94704-0163.

PJM peer review and production are managed by EditFLOW ${ }^{\circledR}$ from Mathematical Sciences Publishers.

\section{PUBLISHED BY}

\section{mathematical sciences publishers \\ nonprofit scientific publishing}

http://msp.org/

(C) 2014 Mathematical Sciences Publishers 


\section{PACIFIC JOURNAL OF MATHEMATICS}

Volume $272 \quad$ No. $1 \quad$ November 2014

Nonconcordant links with homology cobordant zero-framed surgery $\quad 1$ manifolds

JAE CHOON CHA and MARK POWELL

Certain self-homotopy equivalences on wedge products of Moore spaces

Ho Won CHOI and KeE Young LeE

Modular transformations involving the Mordell integral in Ramanujan's lost notebook

Youn-SEO CHOI

The $D$-topology for diffeological spaces

J. DANiEl Christensen, Gordon SinNAMON and ENXIN Wu

On the Atkin polynomials

AHMAD EL-Guindy and Mourad E. H. ISMAIL

Evolving convex curves to constant-width ones by a perimeter-preserving flow

LAIYUAN GaO and Shengliang PAN

Hilbert series of certain jet schemes of determinantal varieties

SudhiR R. GHORPADE, Boyan Jonov and B. A. SeTHuraman

On a Liu-Yau type inequality for surfaces

Oussama Hijazi, Sebastián Montiel and Simon Raulot

Nonlinear Euler sums

ISTVÁN MEZŐ

Boundary limits for fractional Poisson $a$-extensions of $L^{p}$ boundary functions 227 in a cone

LEI QIAO and TAO ZHAO

Jacobi-Trudi determinants and characters of minimal affinizations

STEVEN V SAM

Normal families of holomorphic mappings into complex projective space concerning shared hyperplanes

LiU Yang, Caiyun Fang and Xuecheng Pang 